\title{
Propofol Sedation During Spinal Anaesthesia - A Dose Finding Study
}

\author{
Ghimire A, ${ }^{1}$ Bhattarai B, ${ }^{1}$ Rahman TR, ${ }^{1}$ Singh SN, ${ }^{1}$ Koirala $S,{ }^{1}$ Tripathi $\mathrm{M}^{2}$
}

${ }^{1}$ Department of Anaesthesiology and Critical care, BPKIHS, Dharan, Nepal.

${ }^{2}$ Department of Anaesthesiology and Critical care, SGPGIMS, Lucknow, India

\section{Corresponding Author}

Ashish Ghimire

Department of Anaesthesiology and critical care BPKIHS, Dharan, Nepal

ashishghimi@hotmail.com

\section{Citation}

Ghiimire A, Bhattarai B, Rahman TR, Singh SN, Koirala S, Tripathi M.Propofol sedtion During spinal Anaesthesia- a dose finding study.Kathmandu Univ Med J 2011;35(3):170-3

\begin{abstract}
Background

It is important to be able to measure and maintain a specific sedation level to compare outcomes of different levels of sedation during anesthesia and the aims include general patient comfort, freedom from specific discomfort, and some amnesia for both the block procedure and the surgical operation, in order to meet the patient's preference and safety. In this prospective randomized clinical study, we compared the three different infusion doses of propofol.
\end{abstract}

\section{Objective}

To find out the appropriate infusion dose of propofol for optimal sedation without causing undue side effects in patients undergoing spinal anaesthesia.

\section{Method}

One hundred twenty patients ASA PS I-II were randomly allocated to three groups 1,2 and 3 receiving propofol infusion at the rate of 25,50 and 75 microgram $/ \mathrm{kg} /$ min with concentration of $(0.5 \%),(1 \%)$ and $(1.5 \%)$ respectively. They were observed for sedation score, hemodynamic parameters and satisfaction level. The adverse effects like respiratory depression, nausea and vomiting score were assessed.

\section{Result}

Median sedation score increased in a dose dependent manner, with significantly higher scores in group 2 and 3 compared with group 1 . Hemodynamic parameters were better in group 1 and 2 as judged by mephentermine requirement. The awakening time after stoppage of infusion was significantly delayed in group 3 ( $p$ $<0.001$ ). Respiratory depression, nausea and vomiting were comparable clinically. Almost three fourth of the patients were satisfied with the techniques used.

\section{Conclusion}

Propofol infusion at the rate of $50 \mathrm{mcg} / \mathrm{kg} / \mathrm{min}$ for sedation in spinal anaesthesia provides optimal sedation, early awakening and excellent satisfaction level in the postoperative period

\section{KEYWORDS}

propofol, sedation, spinal anaesthesia.

\section{INTRODUCTION}

Despite increasing use of spinal anaesthesia in anaesthetic practice, anxiety and discomfort still remain important patients concerns. ${ }^{1}$ Adjunctive drugs are used to decrease anxiety, alleviate discomfort, improve hemodynamic stability and induce a feeling of calmness during spinal anaesthesia. However, it is important to be able to measure and maintain a specific sedation level to compare outcomes of different levels of sedation during anesthesia and the aims include general patient comfort, freedom from specific discomfort, and some amnesia for both the block procedure and the surgical operation, in order to meet the patient's preference and safety. ${ }^{2}$ Since regional block is associated with analgesia, a sedative drug should be a good adjunct to allay the anxiety of the patient during surgery. Propofol is a short acting, easily controllable and individually titrable hypnotic and sedative agent. ${ }^{3}$ Because of these pharmacokinetic properties propofol has a quick recovery and has been tried for sedation during spinal anaesthesia with different doses but the exact dose and protocol of propofol use for sedation is debatable. Thus the aim of our study was to find out the optimum propofol infusion dose for sedation without causing undue side effects in patients undergoing spinal anaesthesia. 
Table 1. Demographic Variables, duration of infusion and surgery.

\begin{tabular}{llll} 
Variables & Group 1 $(\mathrm{n}=40)$ & Group 2 $(\mathrm{n}=40)$ & Group 3 (n=40) \\
\hline Age in years (mean+SD) & $38.8 \pm 13.85$ & $42.6 \pm 16.08$ & $40.12 \pm 17.28$ \\
\hline Weight (kg) (mean+SD) & $53.75 \pm 9.44$ & $52.85 \pm 7.54$ & $54.00 \pm 8.06$ \\
\hline Gender (F/M) & $16 / 24$ & $14 / 26$ & $18 / 22$ \\
\hline ASA I/II & $33 / 7$ & $35 / 5$ & $36 / 4$ \\
\hline Duration of infusion (min) (mean+SD) & $71.88 \pm 25.41$ & $68.25 \pm 26.2$ & $65.75 \pm 21.83$ \\
\hline Duration of surgery(min) (mean+SD) & $66.88 \pm 25.41$ & $63.25 \pm 26.2$ & 0.64 \\
\hline
\end{tabular}

\section{METHOD}

One hundred twenty ASA physical status I-II adult patients, scheduled for elective surgery under spinal anaesthesia were studied according to a double-blind protocol approved by our institutional research committee. Patients with a history of allergic reaction to the study medication, chronic opioid or sedative drug use, obesity $(>130 \%$ of ideal body weight), or clinically significant cardiac, hepatic, or renal dysfunction were excluded from participation in this study.

All the patients were premedicated with oral diazepam in the dose of $0.2 \mathrm{mg} / \mathrm{kg}$ the night before and two hours prior to surgery. On arrival in the preoperative holding area, patients were assessed for baseline sedation score and an 18 or 16 gauge intravenous cannula was secured into a large vein on the dorsum of the hand under local anaesthesia.

All patients were given preloading dose $(500 \mathrm{ml})$ of ringer's lactate $30 \mathrm{~min}$ prior to spinal block. We observed for heart rate, non invasive arterial blood pressure (NIBP), respiratory rate $(R R)$, arterial blood saturation by pulse oxymetry (SPO2) and sedation score from one to five scales preoperatively.

To make this study double blinded, three different dilutions of propofol for infusion were prepared in $50 \mathrm{ml}$ syringe with any of the 3 concentrations (0.5\%), (one\%) or $(1.5 \%)$ by consultant anaesthesiologist not involved in the anaesthetic care of the patient. Commercially available propofol ( $2 \%$ ) was diluted with $5 \%$ dextrose to make $(0.5 \%)$,( $1 \%$ and $(1.5 \%)$ solution. Prior to anaesthesia, patients were randomly allocated into three groups of propofol infusion doses. Randomization was done using sealed envelope technique into three groups. All received study drug at the rate of $0.3 \mathrm{ml} / \mathrm{kg} / \mathrm{hr}$ so that dose became $25 \mathrm{mcg} / \mathrm{kg} / \mathrm{min}$, $50 \mathrm{mcg} / \mathrm{kg} / \mathrm{min}$ or $75 \mathrm{mcg} / \mathrm{kg} / \mathrm{min}$ in the three groups 1 , 2 and 3 respectively. Injection lignocaine hydrochloride $40 \mathrm{mg}$ was injected intravenously prior to start of infusion to abolish pain induced by propofol. Propofol was infused through an infusion pump (B-Braun, model 12COF9C9) and was started five minutes prior to spinal anaesthesia and was continued throughout the surgery. Infusion of propofol was discontinued after the wound closure.

All patients were given supplemental oxygen at the rate of two liters / min via a nasal prong. Sedation level was assessed at 15 minutes interval using the sedation score described by Wilson et al as: fully awake and oriented-1, drowsy-2, eyes closed but arousable to command-3, eyes closed but arousable to mild physical stimulation (earlobe tug)-4, eyes closed but unarousable to mild physical stimulation-5. ${ }^{4}$ Hypotensive episodes (MAP<60mmHg) were treated with bolus intravenous fluid and injection mephentermine (3 mg) intravenously. Total requirement of mephentermine was noted. Respiratory depression was scored described by Brodner et al as: normal respiratory rate of 12 to 18 per minute- 1 , respiratory rate $8-12$ breaths per minute-2, respiratory rate $<8$ per minute- $3 .{ }^{5}$ Airway control assessment scored as: no compromise/ no obstruction-1, snoring or noisy breathing-2, assistance required in the form of mask/LMA/ETT-3. Presence of nausea and vomiting were noted till 6 hours and was scored described by Callesen et al: no nausea, no vomiting-1, light nausea, one episode of vomiting-2, moderate nausea, two episode of vomiting-3, severe nausea, three or more episodes of vomiting $-4 .{ }^{6}$ Nausea and vomiting were treated with intermittent bolus injection ondansetron $4 \mathrm{mg}$ intravenously.

In the postoperative period, we enquired whether the patients were satisfied with the technique used and would like to be similarly sedated in future for similar surgeries or not.

Parametric and non parametric data were collected and were entered in a master chart in MS Excel program. Data were analyzed using the statistical package for social science (version 11.5 for Windows, SPSS Inc. Chicago University, USA). The significant difference of mean between the intervention groups was calculated using ANOVA test. For the discrete variables, chi square test was used. For all the purposes p-value of less than 0.05 was considered significant.

\section{RESULT}

Demographic data and duration of infusion and surgery for the three treatment groups are summarized in Table 1. The three groups did not differ with respect to age, weight, sex and ASA physical status. Similarly the total duration of infusion of propofol and surgery were comparable among the groups. (Table 1)

Hernioplasty was most frequent surgery in our study, followed by lower limb orthopedic surgeries. (Table 2)

Baseline sedation scores were comparable between the groups (figure 1). Median sedation score recorded upon 
Table 2. Comparison of types of surgeries.

\begin{tabular}{|llll|}
\hline Types of surgery & $\begin{array}{l}\text { Group 1 } \\
(\mathbf{n = 4 0 )}\end{array}$ & $\begin{array}{l}\text { Group 2 } \\
(\mathbf{n = 4 0 )}\end{array}$ & $\begin{array}{l}\text { Group 3 } \\
(\mathbf{n = 4 0 )}\end{array}$ \\
\hline Hernioplasty & 18 & 20 & 22 \\
\hline Lower limb orthopedic surgery & 12 & 9 & 12 \\
\hline Vesicle calculus & 5 & 5 & 3 \\
\hline Vaginal hysterectomy & 0 & 4 & 1 \\
\hline Skin graft of lower limbs & 1 & 1 & 2 \\
\hline Trans-abdominal hysterectomy & 3 & 0 & 0 \\
\hline Penile amputation & 1 & 1 & 0 \\
\hline
\end{tabular}

\section{Median Sedation Score}

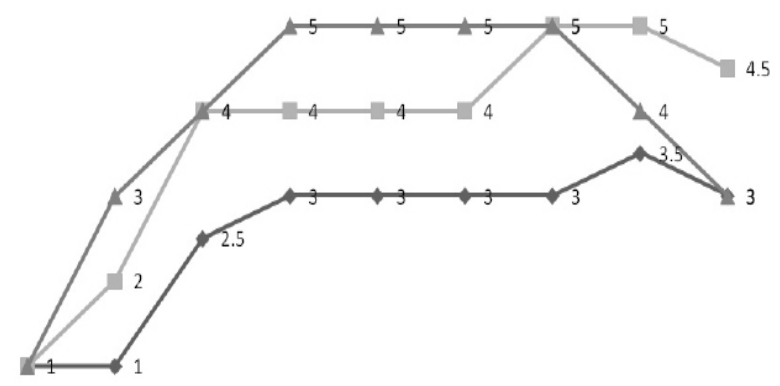

Baseline $15 \mathrm{Min} \quad 30 \mathrm{Min} \quad 45 \mathrm{Min} \quad 60 \mathrm{Min} \quad 75 \mathrm{Min} \quad 90 \mathrm{Min} \quad 105 \mathrm{Min} \quad 120 \mathrm{Min}$ $\multimap$ Group1 -G-Group2 $\leftarrow$ Group3

Figure 1. Comparison of median sedation score.

arrival in the operation theater did not differ significantly among the groups. Median sedation score increased in a dose dependent manner, with significantly higher scores in group 2 and 3 compared with group 1 . Sedation score after 15 minutes of starting of infusion up to 75 minutes were significantly different among the groups. Median sedation score remained 3 in group 1, 4 in group 2 and 5 in group 3. However sedation scores at 105 and 120 minutes were not significantly different among the groups.

The awakening time after stoppage of infusion was delayed significantly in group $3(6.85 \pm 3.49)$ as compared to group $1(2.85 \pm 2.63)$ and $2(4.78 \pm 3.18)$. Time to spontaneous opening of eyes were significantly different among the groups $(p<0.001)$.

There was no incidence of nausea, vomiting and respiratory depression in group 1 . One patient in group 2 had 1 episode of nausea and vomiting and respiratory rate less than 12 per minute. Two patients in group 3 had respiratory rate less than 12 per minute. Patients belonging to group 1 had significantly less incidence of hypotension (10\%) compared to group $2(27.5 \%)$ and $3(50 \%)$. $(p<0.006)$

All patients received supplemental oxygen throughout the procedure and in recovery area and SPO2 was maintained ( $\geq 98 \%$ ).

Significantly higher number of patients in group 2 (90\%) and 3 (95\%) expressed satisfaction in comparison to group $1(50 \%) .(p<0.001)$

\section{DISCUSSION}

Our study has shown that propofol infusion at the rate of $50 \mathrm{mcg} / \mathrm{kg} / \mathrm{min}$ in patients undergoing surgery under subarachnoid block anaesthesia is associated with the most desirable outcome compared to $25 \mathrm{mcg} / \mathrm{kg} / \mathrm{min}$ and $75 \mathrm{mcg} / \mathrm{kg} / \mathrm{min}$. Mackenzie and Grant have also reported optimal sedation with a mean infusion rate of $3 \mathrm{mg} / \mathrm{kg} /$ $\mathrm{hr}(50 \mathrm{mcg} / \mathrm{kg} / \mathrm{min})$ but in patient over age of 65 . Younger patient in their study required higher infusion rate. ${ }^{3}$

Because of the variability of individual reactions to sedatives, a dose protocol cannot be used to assess sedation. Observer-based sedation scales offer a quick and easily administered means of assessing the level of sedation, provided that they demonstrate high inter-rater reliability and fulfill other criteria for construct validity. ${ }^{7}$

Smith et al. in their study observed that the patients receiving loading dose of propofol and infusion at the rate of $67 \mathrm{mcg} / \mathrm{kg} / \mathrm{min}$ were over sedated (i. e. unresponsive to verbal command), but, it is difficult to compare our study with theirs since their study designs and the sedation score used were different. ${ }^{8}$

In our study, awakening, which was assessed as spontaneous eye opening after the stoppage of propofol infusion was significantly delayed by 4 minutes in the group $75 \mathrm{mcg} /$ $\mathrm{kg} / \mathrm{min}$ as compared to the group receiving infusion at $25 \mathrm{mcg} / \mathrm{kg} / \mathrm{min}$. Patients receiving propofol infusion at the rate of $50 \mathrm{mcg} / \mathrm{kg} / \mathrm{min}$ were awake in 5 minutes which is comparable to the awakening time reported in the group receiving propofol infusion at the rate of $3 \mathrm{mg} / \mathrm{kg} / \mathrm{hr}$ in the study by Mackenzie and Grant. ${ }^{3}$

Expectedly more patients in $75 \mathrm{mcg} / \mathrm{kg} / \mathrm{min}$ group in our study required more fluid and mephentermine to maintain normal blood pressure despite preloading in all patients.

In the present study three patients had respiratory rate less than 12 after 30 minutes of infusion, two of them from group $75 \mathrm{mcg} / \mathrm{kg} / \mathrm{min}$ and one from group $50 \mathrm{mcg} / \mathrm{kg} / \mathrm{min}$. All of them had higher spinal block upto T4-5 which probably contributed to the lower respiratory rate. Reduction in ventilatory response has been reported in patients receiving propofol infusion for sedation. ${ }^{9,10}$ However, the respiratory depression occurring after infusion of propofol for sedation in our study was not associated with hypoxia as reported in other study. ${ }^{11}$ Use of supplemental oxygen in our study must have prevented hypoxia.

One of the patients from group two had one episode of nausea and vomiting, hypotension and respiratory rate less than 12 during intraoperative period and was treated with intravenous bolus fluid, ondansetron and mephentermine. The reported incidence of nausea and vomiting after propofol infusion is generally low, and an antiemetic effect has been suggested. ${ }^{12,8}$

In this study, almost three fourth of the patients were satisfied with the techniques used and would like to be 
sedated similarly for similar future surgery. Sedation has been shown to increase patient satisfaction during regional anaesthesia. ${ }^{13}$ In one study more than (90\%) patients receiving propofol or midazolam-fentanyl sedation preferred sedation rather than general anaesthesia for future surgeries, ${ }^{14}$ although patients may be generally satisfied whatever they are offered..$^{15}$

The present study suffers some limitations which include not using objective monitoring of the sedation level with bispectral index and monitoring of plasma propofol concentration.

\section{CONCLUSION}

Propofol infusion at the rate of $50 \mathrm{mcg} / \mathrm{kg} / \mathrm{min}$ during spinal anaesthesia provides optimal sedation, less hemodynamic instability in the intraoperative period, early awakening from sedation and excellent satisfaction level in the postoperative period.

\section{REFERENCES}

1. Bhattarai B, Rahman TR, Sah BP, Singh SN. Central neural blocks: a quqlity assessment of anaesthesia in gynaecologocal surgeries. Nepal Med Coll J 2005; 7(2) ; 93-96.

2. Hohener D, S. Blumenthal, A. Borgeat. Sedation and regional anaesthesia in the adult patient. Br J Anaesth 2008;100 (1): 8-16.

3. Mackenzie N, Grant IS. Propofol for intravenous sedation. Anaesthesia 1987;42:3-6.

4. Wilson E, David A, Mackenzie N, Grant IS.Sedation during spinal anaesthesia: Comparison of Propofol and Midazolam. Br J Anaesth 1990; 64:48-52.

5. Brodner G, Mertes N, Van Aken H, Pogatzki E, Burekle H,Marcus M, Mollhoff T. Epidural analgesia with local anaesthetics after abdominal surgery: Earlier motor recovery with $0.2 \%$ ropivacaine than $0.175 \%$ bupivacaine. Anaesth Analg 1999; 88:128-33.

6. Callesen T, Schouenborg L, Nielsel D, Gludager H,Kehlet $H$. Combined epidural-spinal opioid -free anaesthesia and analgesia for hysterectomy. Br J Anaesth 1999;82:881-5.

7. Nemethy et al. Assessing Sedation with Regional Anesthesia: InterRater Agreement on a Modified Wilson Sedation Scale. Anesth Analg 2002;94:723-8.

8. Smith I, Monk TG, White PF, Ding Y Propofol infusion during regional anaesthesia: Sedative amnestic and anxiolytic properties. Anesth Anal 1994; 79:312-39.

9. Blouin RT, Seifert HA, Babenco HD, Conard PF, Gross JB.Propofol depresses the hypoxic ventilatory response during conscious sedation and isohypercapnia. Anesthesiology 1993; 79:1177-82.

10. Nieuwenhuijs D, Sarton E, Teppema L, Dahan A. Propofol for monitored anaesthesia care: implications on hypoxic control of cardiorespiratory responses. Anesthesiology 2000; 92: 46-54.

11. Rosa G, Conti G, Orsi O: Effect of low dose Propofol administration on central respiratory drive, gas exchanges and respiratory pattern. Acta Anaesthesiol Scand 1992; 36:128-31.

12. Borgeat A, Wilder-Smith $\mathrm{OH}$, Saiah M, Rifat K. Subhypnotic doses of propofol possess direct antiemetic properties. Anesth Analg 1992; 74: 539-41.

13. Wu CL, Naqibuddin M, Fleisher LA. Measurement of patient satisfaction as an outcome of regional anesthesia and analgesia: a systematic review. Reg Anesth Pain Med 2001; 26:196-208.

14. Hasen KV, Samartzis D, Casas LA, Mustoe TA. An outcome study comparing intravenous sedation with midazolam/fentanyl (conscious sedation) versus propofol infusion (deep sedation) for aesthetic surgery. Plast Reconstr Surg 2003; 112: 1683-9.

15. Kryger ZB, Fine NA, Mustoe TA. The outcome of abdominoplasty performed under conscious sedation: six-year experience in 153 consecutive cases. Plast Reconstr Surg 2004; 113: 1807-17. 